\title{
Kernos
}

Revue internationale et pluridisciplinaire de religion grecque antique

7| 1994

Varia

\section{Syncrétismes et cultes indigènes en Asie Mineure méridionale}

\section{René Lebrun}

\section{OpenEdition \\ Journals}

\section{Édition électronique}

URL : http://journals.openedition.org/kernos/1103

DOI : 10.4000/kernos. 1103

ISSN : 2034-7871

\section{Éditeur}

Centre international d'étude de la religion grecque antique

\section{Édition imprimée}

Date de publication : 1 janvier 1994

ISSN : 0776-3824

\section{Référence électronique}

René Lebrun, « Syncrétismes et cultes indigènes en Asie Mineure méridionale », Kernos [En ligne], 7 | 1994, mis en ligne le 20 avril 2011, consulté le 19 avril 2019. URL : http://journals.openedition.org/ kernos/1103 ; DOI : 10.4000/kernos. 1103 


\section{SYNCRÉTISMES ET CULTES INDIGÈNES}

\section{EN ASIE MINEURE MÉRIDIONALE}

En nous limitant dans le temps à la période gréco-asianique et dans l'espace au sud de l'Asie Mineure, nous tenterons de relever des cas typiques à rattacher soit à quelque forme de syncrétisme total ou partiel, soit à des tentatives d'assimilation, soit à une résistance cultuelle. D'emblée, il importe de signaler combien, en de nombreuses régions de Lycie, de Pamphylie, de Pisidie, de Lycaonie et de Cilicie, la tradition religieuse anatolienne remontant au second millénaire avant J.-C. s'est maintenue vivante sous sa forme louvite face à un hellénisme envahissant, en particulier sur les côtes; n'oublions cependant pas la présence mycénienne en plusieurs points du littoral égéen et méditerranéen dès la seconde moitié du second millénaire.

\section{Syncrétismes (partiels), assimilations}

Plusieurs personnalités divines rencontrées dans les inscriptions grecques illustrent des formes d'assimilation ou de syncrétisme qu'il faut essayer d'évaluer.

1. Zeus

À l'exception des cas où Zeus était adoré par d'authentiques Grecs d'Asie Mineure, la majorité des Zeus mentionnés dans les inscriptions locales représente en réalité la continuité d'un dieu louvite de l'orage à identifier à Tarbunt. Zeus est un déguisement appellatif grec donné à un dieu de l'orage local de tradition anatolienne, cette dénomination étant facilitée par une identité approximative de la fonction. Il y a donc ici une forme de syncrétisme, à tempérer toutefois par la constatation que l'iconographie de ces Zeus reste indigène, étrangère à toute influence grecque. De plus, le nom de Zeus est régulièrement suivi du toponyme auquel le dieu de l'orage se rattache, ce qui confirme son caractère local; ainsi, Zeus Korikios, Olbios, Tarsios. Ce sont aussi des épiclèses manifestement asianiques qui peuvent accompagner dans 
l'inscription le nom de Zeus ${ }^{1}$. Comme nous l'avons déjà souligné, tous ces Zeus cachent en fait le dieu louvite Tarbunt. Remarquons que le théonyme louvite du dieu de l'orage subsiste dans le lycien Trqqñt, souvent mentionné dans l'épigraphie lycienne ou dans l'anthroponymie gréco-asianique sous la forme Tó $\rho \kappa \omega v$ ou Tó $\rho \chi \omega v$, Tpók $\omega v$. Si la forme louvite grécisée du nom est bien présente dans les anthroponymes théophores, le théonyme *Tó $\rho \chi \omega v$ n'a pas encore été trouvé.

\section{Athéna}

Avec Athéna, nous sommes en présence d'un bon exemple de syncrétisme, ou plus exactement de syncrétisme ou d'assimilation partiels. En effet, il n'y a pas lieu de reconnaître spontanément la déesse athénienne comme telle. En fait, sous la pression de la grécisation, plusieurs déesses poliades (d'anciennes déesses-reines) se dénommèrent Athéna dans les inscriptions grecques, mais elles n'en correspondent pas moins à une profonde réalité asianique-louvite. Le cas lycien est significatif; les documents assurent plusieurs fois l'équation Athéna = Maliya lycienne, laquelle constitue la fidèle continuité de la déesse Maliya des textes hittites et louvites du second millénaire; Maliya était importante dans tout le panthéon local en tant que divinité protectrice des prairies, des vergers, des sources ${ }^{2}$.

\section{Hermès}

Particulièrement vénéré en Cilicie - par exemple à Elaioussa, Korykos, Olba, Diocésarée ou Hamaxia d'après les inscriptions, les monnaies et même des indications littéraires, notamment dans les Halieutica d'Oppien -, Hermès y est présenté comme un $\theta \varepsilon o ̀ \varsigma ~ \pi \alpha \tau \rho \hat{o} ం \varsigma$

1 Ainsi Zzù Osogoa, cf. R. Lebrun, A propos des déesses Maliades et de quelques épiclèses gréco-asianiques, in Kernos, 2 (1989), p. 85-86.

2 Voir R. LEBRUN, Maliya, une divinité anatolienne mal connue, in J. Quaegebeur (éd.), Studia Paulo Naster Oblata, II : Orientalia Antiqua, Leuven, 1982 (Orientalia Lovaniensia Analecta, 13), p. 123-130. Notons l'importance du fragment de vase en argent représentant le jugement de Pâris; pour chacun des trois personnages représentés, on trouve l'inscription du nom en lycien : Pedrita (Aphrodite), AliXssa[- (Alexandre) et Mal[iya (Athéna), cf. D.E. STrong, A Greek Silver Head-Vase, in British Museum Quartely, 28 (1964), p. 95-102; R.D. BARNETT, A Silver-Head Vase with Lycian Inscriptions, in Mansel'e Armagan, II, 1974, p. 893-903. 
très important à Korykos ${ }^{3}$. Avec de bonnes raisons, $\mathrm{Ph}$. Houwink ten Cate estime que le dieu Hermès cacherait le grand dieu louvite Kurunta aboutissant à Runta au premier millénaire, soit un dieu protecteur de la nature sauvage (sumérogramme ${ }^{\mathrm{d}} \mathrm{KAL}$ ou $\mathrm{d}_{\mathrm{LAMMA}}{ }^{4}$. Tout comme Héraklès, Hermès est à considérer en Asie Mineure comme un dieu fourre-tout. Par un de leurs aspects, fût-il mineur, ces dieux peuvent être assimilés à un dieu local anatolien. Certaines dénominations divines grecques y sont utilisées, non avec leurs valeurs grecques, mais dans la ligne d'une tradition conforme à celle de l'emploi des sumérogrammes au second millénaire 5 . Il nous faut encore signaler un nombre appréciable de théophores en $\mathrm{P} \omega \vee \delta(\alpha)$-, par exemple P $\omega \vee \delta \alpha \varsigma, P \omega v \delta \varepsilon \rho \beta \varepsilon \mu \iota \varsigma$,

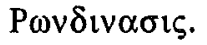

\section{Héraklès}

Le cas d'Héraklès est donc analogue à celui d'Hermès, étant entendu qu'il s'agit du dieu Héraklès vénéré en tant que divinité importante (cf. Thasos) et non de la figure du héros grec habituellement retenue. Ainsi, il est un des grands dieux de Tarse avec Persée, Apollon et Athéna ${ }^{6}$; il est même qualifié de dieu fondateur, ce qui ne peut constituer une référence à une fondation grecque vu la très haute antiquité des origines de Tarse. Il se peut que, lorsqu'ils accréditaient

3 Cf. G.DAGRon et D.FeISSEL, Inscriptions de Cilicie, Paris, 1987 (Travaux et mémoires du centre de recherche d'histoire et civilisation de Byzance, Collège de France, Monographies, 4), p. 44-46; on y trouve notamment l'étude de l'inscription figurant sur un autel de Korykos : Hermès y est défini comme un

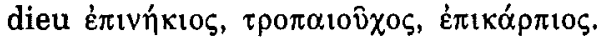

4 Ph. Houwink ten Cate, The Luwian Population Groups, Leyde, 1961, p. 212 213.

5 Ainsi, dans les textes hittites et louvites, ${ }^{\mathrm{d}} \mathrm{IM}$ ne désigne pas le dieu de l'orage sumérien mais un dieu de l'orage anatolien (hatti, hittite, louvite ou hourrite selon les lieux et les époques). Il convient encore de se demander si, sur plusieurs inscriptions d'Anatolie méridionale, notamment de Lycie, le thème ERME- renvoie automatiquement à Hermès; ne pourrait-il s'agir du maintien de la forme louvito-lycienne du nom de la lune (louvite arma - = lycien erme); ainsi, sur le relief d'Alifahrettin (cf. L. ROBERT, Documents d'Asie Mineure,

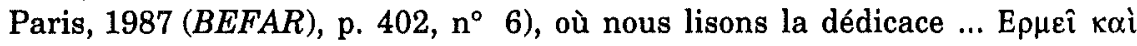
$\Delta$ tookópors accompagnant le relief typique en ces régions d'une déesse, normalement lunaire, entourée de deux cavaliers se faisant face et assimilés

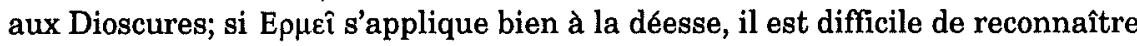
dans ce nom le dieu Hermès.

6 Voir le premier discours de Dion de Pruse. 
les légendes argiennes relatives à leurs origines, les Tarsiotes n'avaient plus une véritable conscience de ce qu'étaient leurs ancêtres, notamment au second millénaire, à savoir des Louvites en majorité. Cette inconscience était renforcée par la nécessité qu'éprouvèrent de nombreuses cités d'Asie Mineure de codifier des traditions légendaires hellénisantes - surtout argiennes et spartiates - lorsqu'elles durent fournir les preuves documentées de leurs origines grecques pour participer à la Confédération du Panhellénion fondée à Athènes par Hadrien ${ }^{7}$. Les lignes directrices de ces légendes se formèrent peut-être dès les premiers contacts entre colons grecs et indigènes d'Asie. Ceci ne concernait toutefois que les lettrés des villes et non les populations campagnardes fidèles aux traditions indigènes. Toujours est-il que le dieu louvite Tarbunt est à reconnaître dans le Zeus Tarsios ou Tarsènos désigné aussi comme $B^{\prime} l \operatorname{Tr} z$ (araméen) durant l'empire achéménide. En dessous du dieu de l'orage, nous voyons dans le panthéon tarsiote du second millénaire le redoutable dieu Santa qui se retrouve tel quel avec sa dénomination indigène à l'époque gréco-asianique dans le nom $\Sigma \alpha v \delta \omega v$, le dieu au bûcher identifié à Héraklès ou à Melqart ${ }^{8}$. Ces divers noms rencontrés à la période gréco-romaine étaient certes liés aux composantes ethniques de la cité. Il nous faut par ailleurs relever l'assimilation d'Héraklès au dieu lycien-pisidien Kakasbos, le dieu cavalier et guerrier à la massue, vêtu d'une chlamyde, parfois d'une tunique ${ }^{9}$. L'assimilation de Kakasbos à Héraklès est particulièrement attestée à Telmessos et sur le haut plateau pisidien ${ }^{10}$.

\section{La triade Hélène et les Dioscures}

Ce "thème" est évoqué plusieurs fois par les reliefs rupestres de Lycie et de Pisidie. Surmontée du croissant lunaire, une déesse habillée

7 Cf. L. ROBERT, in $B C H, 107$ (1983), p. 564.

8 E. LAROCHE, Un syncrétisme gréco-anatolien : Sandas = Héraklès, in Les syncrétismes dans les religions grecque et romaine, Paris, 1973, p. 103-114.

9. Pour Kakasbos, voir l'étude de L. RoBERT, Un dieu anatolien : Kakasbos, in Hellenica, 3 (1946), p. 38-74; théonyme composé de -asbos qui renvoie au lycien esbe - "cheval». On complétera par L. ROBERT, Documents d'Asie Mineure, Paris, 1987, p. 76, 392, 398, 400-404, 413, 416-417, 423, 434, 437-438.

10 H. METZGER, Catalogue des monuments votifs du musée d'Adalia, Paris, 1952, p. 13-16; L. RoBert, Documents d'Asie Mineure, p. 76, 392, 398, 400. Notons encore que les récentes fouilles entreprises à Sagalassos laissent supposer que Kakasbos aurait été un dieu particulièrement vénéré dans la cité. 
est entourée de deux cavaliers qui s'affrontent; les inscriptions identi-

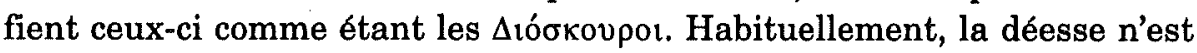
pas nommée, comme si la désignation de celle-ci allait de soi, et les numismates comme les archéologues (notamment Chapouthier et Perdrizet) ont unaniment franchi le pas. Dans la foulée de la fondation prétendue de Tarse par les Argiens, l'origine spartiate du culte de cette triade ne faisait aucun doute et tout naturellement on songeait à Hélène entourée des Dioscures. Il n'en est rien!

En fait, le thème de notre triade se trouve souvent représenté dans les campagnes en des lieux d'accès difficile, tenus à l'écart des grands axes suivis par la divulgation de la pensée hellénique. La manière dont les Dioscures pisidiens sont représentés n'est pas grecque; en l'occurrence, il ne sont pas nus. Une seule fois, la déesse est identifiée comme étant Artémis ${ }^{11}$. La déesse revêt donc ici un caractère lunaire. Nous avons, avec la représentation de la triade, l'image des dieux du paysan d'Anatolie méridionale qu'il grave à même le rocher, selon l'antique tradition anatolienne : une déesse lunaire, par ailleurs protectrice de la nature sauvage, entourée de deux dieux protecteurs ou guerriers. Les indigènes ont emprunté à la Grèce les noms, pas la réalité; il n'existe rien de commun entre Hélène et les Dioscures spartiates et nos reliefs.

\section{Arès}

Arès, le redoutable dieu de la guerre, a laissé peu de traces de son culte en Grèce même; ses lieux de culte, les inscriptions rédigées en son honneur y sont rares. Au plan littéraire, on le retrouve dans l'épopée homérique et la tragédie attique, mais l'image donnée dans ces œuvres paraît peu conforme aux témoignages cultuels ${ }^{12}$. Le culte d'Arès semble toutefois avoir été plus florissant en dehors du continent grec, en Asie Mineure notamment. Plusieurs cités conservaient des stèles, des autels qui lui étaient consacrés; le monnayage de plusieurs d'entre elles était à l'effigie $\mathrm{du}$ dieu. Une épiclèse typiquement asianique accompagne parfois le nom du dieu; d'autre part, l'analyse des symboles liés au dieu doit toujours être minutieuse. Un bon exemple est l'autel d'Arès Kiddeudas (épiclèse asianique) trouvé en Pisidie; il s'agit, certes, du dieu guerrier local, mais il est simultanément un dieu agraire

11 Cf. le relief de Macun Asart = L. RoBert, Documents d'Asie Mineure, p. 404,

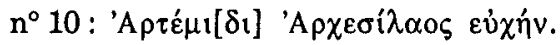

12 F. JoUAN, Le dieu Arès : figure rituelle et image littéraire, in Le point théologique, 52 (1989), p. 125-140. 
karpodotès comme l'indiquent la corne d'abondance et la grappe de raisins sculptées sur un des flancs de l'autel ${ }^{13}$. Comme l'a justement observé Louis Robert, le dieu Arès a presque toujours constitué dans le Sud de l'Asie Mineure le déguisement grec d'un dieu guerrier indigène; celui-ci pouvait être parfois affecté d'un caractère supplémentaire, comme l'Arès Kiddeudas ${ }^{14}$.

\section{Mâ}

Le cas de Mâ présente une situation inverse de celle évoquée pour les dieux «grecs» précédemment étudiés; nous observons ici une déesse d'Anatolie dont le culte va se propager en Grèce et à Rome, faisant l'objet d'une forme de syncrétisme ou d'assimilation. Mâ était la grande déesse de Comana de Cappadoce (Kummanni, grand centre religieux au second millénaire) et de Comana du Pont. Strabon écrit en XII, 2, 3 :

Dans le massif de l'Anti-Taurus se trouvent des vallées étroites et profondes. C'est là que sont situés Comana et le sanctuaire d'Enyô connu là-bas sous le nom de sanctuaire de Mâ. Comana est une ville considérable, mais sa population se compose en majeure partie des théophorètes et du personnel servile du temple.

Mâ est attestée dans l'anthroponymie gréco-asianique; il s'agit d'une formation primaire résultant peut-être de la simplification du nom divin hatti Mamma ${ }^{15}$. Ce nom évoquerait la «Magna Mater» du lieu. Il est possible que, durant la seconde partie du deuxième millénaire, il y ait eu un syncrétisme entre Mamma/Mâ et la déesse hourrite Hébat (à l'origine une Mater de l'Ouest syrien devenue l'épouse du dieu de l'orage hourrite Teshub et assimilée plus tard à la grande déesse Soleil d'Arinna, l'épouse du dieu de l'orage du Hatti), divinité dont le culte, comme celui de Teshub, était particulièrement important à Kummanni = Comana. Avec la disparition de l'empire hittite et de la langue hourrite qui lui est liée, l'ancienne dénomination de la déesse réapparaîtrait, comme ce fut le cas en d'autres lieux; elle aurait en même temps hérité du caractère solaire de Hébat. À l'appui de cette hypothèse, retenons une effigie de Mâ, datant de l'époque grécoasianique, où elle apparaît en costume asianique, entourée de deux aigles, la tête nimbée et radiée. Son culte a pu évoluer vers une certaine

13 Cf. L. ROBERT, Documents d'Asie Mineure, p. 424-427.

14 Cf. L. ROBERT, Documents d'Asie Mineure, p. 423.

15 Cf. E . LAROCHE, Les noms des Hittites, Paris, 1966, p. 240. 
brutalité lors de l'assimilation à l'italique Bellone (rappelons que Sylla introduisit son culte à Rome) et à la grecque Enyô ${ }^{16}$.

\section{Cas de résistances}

Certains dieux liés à une cité ou à une région de l'Asie Mineure méridionale ne se sont prêtés à aucun syncrétisme. Le théonyme anatolien a ainsi subsisté, que le dieu soit mentionné dans des documents en langue indigène ou en langue grecque. Il arrive aussi que le nom du dieu ait fait l'objet d'une traduction grecque. Voici quelques exemples significatifs :

\section{a) Dieux cariens}

1. Sinuri : le grand dieu de Mylasa, vénéré également à Hyllarima, était manifestement un dieu de l'orage carien se rattachant à la tradition anatolienne; son iconographie de typologie indigène ainsi que sa dénomination ne laissent aucun doute à ce sujet. Jamais Sinuri n'est assimilé à Zeus; il conserve toujours son nom. La position peu accessible des sanctuaires du dieu, tenus à l'écart des grands axes de circulation ou de pénétration en Asie Mineure, peut expliquer le maintien sans partage d'un culte national ${ }^{17}$.

2. Arkésima et Basileus Kaunios:Basileus Kaunios (lycien Xñtawati Xbideñni), grand dieu d'une cité carienne proche de la Lycie, nous était déjà connu par un passage du pilier xanthien, datable de la fín du Ve siècle av. J.-C., où il faisait partie d'une triade avec Maliya et Ertemi $=$ Artémis $^{18}$. Un culte carien était déjà implanté à ce moment dans la cité sanctuaire de Létô. La découverte en 1973 d'une stèle trilingue, toujours à Xanthos, a permis de déterminer qu'en 358, sous Pixodaros, satrape de Carie et de Lycie, le culte du "Roi de Caunos" se

16 Voir H. SEYRIG, Scripta Varia, Paris, 1985 (Institut français d'archéologie du Proche-Orient, Bibliothèque archéologique et historique, 125), p. 705-707. Notons que d'après TAM V, Vienne, 1989, une inscription dédicatoire d'Hyrkanis ( $\left.\mathrm{n}^{\circ} 1305\right)$ atteste un culte de Mâ Anikètos en Lydie occidentale.

17 Pour le dieu Sinuri, voir L. ROBERT, Le sanctuaire de Sinuri près de Mylasa, Première partie : Les inscriptions grecques, Paris, 1945 (Mémoires de l'Institut Français d'Archéologie de Stamboul, 7), 119 p. et 15 pl.; A. LAUMONIER, Les cultes indigènes en Carie, Paris, 1958, p. 175-183, 458-459.

Cf. $T L$ ( = TAM I) 44 c 7-9. 
trouva renforcé dans la capitale lycienne ${ }^{19}$. La dénomination grecque du dieu est une simple traduction du lycien Xñtawati Xbideñni, appellation fonctionnelle soulignant le caractère éminent du dieu ${ }^{20}$. Cette trilingue xanthienne nous a fait découvrir aussi un nouveau dieu carien, associé à Basileus Caunios : lycien Arkkazuma reproduit en

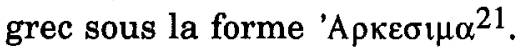

\section{b) Dieux lyciens}

1. Tobaloas et Trôsobios: Tobaloas, $\theta$ eò Ernes) ainsi que Trôsobios (lyc. Trzzubi), dieu de Limyra (lyc. Zémuri), portent manifestement des noms anatoliens, mais ceux-ci nous sont connus essentiellement sous leur forme grécisée; leur cas est semblable à celui de Sinuri ${ }^{22}$.

2. Les nymphes et Eleuthéra : le culte des nymphes, divinités des sources, se rencontre partout en Lycie. Elles sont tantôt trois, tantôt neuf, et se présentent aussi, à l'époque gréco-romaine, comme musiciennes. La stèle trilingue de Xanthos (lyc. Arñna = "source») nous apprend que le lycien Eliyãna est traduit en grec par Nó $\mu \varphi \alpha \iota^{23}$. Quant à Eleuthéra,

19 Pour le texte lycien, cf. E. LAROche, La stèle trilingue du Létôon de Xanthos : le texte lycien, in CRAI (1974), p. 115-225; E. LAROCHE, La stèle trilingue du Létôon : version lycienne, in Fouilles de Xanthos, 6, Paris, 1979, p. 104 sq. Pour la version grecque, ef. H. METZGER, in CRAI (1974), p. 132-149. Une publication des trois versions avec compléments bibliographiques et désignation numérotée de cette trilingue au sein du corpus lycien (= N 320) se trouve dans G. NeumanN, Neufunde lykischer Inschriften seit 1901, Vienne, 1979, p. 44-47.

20 En effet, le lycien $X \tilde{n}$ tawat(i)-continuant le louvite bandawat(i)- répond au grec $\beta \alpha \sigma \curlywedge \lambda \varepsilon v ́ \xi ;$ Xbidẽ ñni- est l'adjectif ethnique lycien dérivé de Xbide = Caunos.

21 Cf. l'inscription N 320 version lycienne 1. 18, 24; version grecque 1. 16, 23; version araméenne 1.8 où malheureusement le nom divin araméen ou

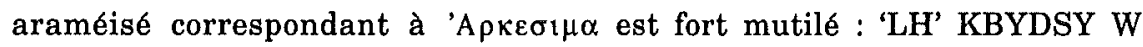
R/K/D/N ?[, cf. A. DUPONT-SOMMER, in CRAI (1974), p. 144-145. Notons que le dieu Arkésima nous est connu par deux décrets hellénistiques et une dédicace de l'île de Cos, cf. G. BEAN, in $J H S, 74$ (1974), p. 95, n' 37 b, et p. 98, n 38 c; L. ROBERT, in Bulletin épigraphique, 1956, 274 c; W.R. PATON et E.L. HICKs, The Inscriptions of Cos, Oxford, 1891, p. 109, n 53.

22 La forme lycienne du théonyme est mentionnée deux fois dans l'inscription $T L$ 111, 1. 1 et 4. Pour Tobaloas et Trôsobios, voir entre autres R. LEBRUN, Problèmes de religion anatolienne, in Hethitica, 8 (1987), p. 244, 246.

23 Cf. $\mathrm{N} 320$ l. 40 du texte lycien = 1. 34-35 du texte grec; pas d'équivalent dans la version araméenne. H. EICHNER, in $O r, 52$, p. 62-64, propose pour le nom lycien une étymologie : <*ali- $\mathrm{h}_{3}$ on- "ayant de l'eau". 
grande déesse de Myra, vénérée également à Kyanéai, nous savons par une glose de Stéphane de Byzance que son nom indigène était 'E qui renvoie au hittite-louvite arawa-«libre»; l'appellation grecque est donc une simple traduction du lycien. La déesse 'E $\lambda \varepsilon v i \theta \varepsilon \rho \alpha$ est bien représentée sur les monnaies, notamment sur celles frappées sous Gordien, si importantes pour l'histoire des religions : on y remarque deux hommes brandissant une double hache et repoussés d'un arbre par deux serpents; entre les branches se distingue l'idole de la déesse ${ }^{24}$.

3. Qebeliya et Qeli : voici deux divinités attestées uniquement dans les inscriptions en langue lycienne; elles offrent un bon exemple de continuité cultuelle et de résistance à toute forme d'hellénisation par voie de syncrétisme ou, jusqu'à preuve du contraire, d'assimilation. Qebeliya, déesse de Limyra, continue le hittite-louvite Hapaliya et doit représenter une divinité Fleuve ${ }^{25}$. S'il n'existe aucune forme grécisée de ce théonyme, en revanche il est présent dans des toponymes tels que Kabalis (ville entre la Lycie et la Pamphylie) ou la Kabalide. Quant à Qeli, il pourrait certes remonter, d'après l'évolution phonétique du louvite vers le lycien, au louvite hali-«jour» et il existe, bien sûr, au second millénaire une divinité désignée comme "le Jour favorable"; toutefois, tenant compte de la possible disparition de la gutturale dans le groupe liquide + gutturale en position interne, il est permis de reconnaître dans Qeli l'aboutissement du hittite-louvite Halki la (déesse) Grain, une des divinités importantes pour le paysan hittite ${ }^{26}$. Le lycien Qeli doit peut-être se voir rattaché à $K \varepsilon \lambda_{\imath}$ débutant plusieurs noms propres $^{27}$.

24 Pour le théonyme 'E $\rho \varepsilon v \alpha$ et son rapport avec arawa-et Eleuthéra, cf. J. PuHvel, Hittite Etymological Dictionary, vol. 1, 1984, p. 120. Pour la représentation d'Eleuthéra sur les monnaies, cf. L. R овERT, Hellenica X, Paris, 1955, p. 198-199.

25 Qebeliya : TL 102, 3; la forme résulte tout naturellement du louvite Hapaliya à décomposer en hapa - "rivière» + le suffixe d'appartenance - li -, cf. G. NEUMANN, in Die Sprache, 20 (1974), p. 109.

26 Cf. N 3221.2 où le constructeur du tombeau est qualifié de qelehi kumaza = "prêtre de Qeli». G. NEUMANN, in Florilegium Anatolicum (Fs E. Laroche), Paris, 1979, p. 270, voit dans Qeli la continuité du hittite-louvite Halki, opinion assortie néanmoins d'une réserve.

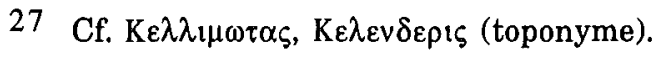




\section{c) Deux cas difficiles : Kubaba et Létô}

1. Kubaba : Grande déesse de Kargamish (Magna Mater) depuis une haute antiquité, en raison de la promotion de sa cité au rang de seconde ville de l'empire hittite, elle fut vénérée en Anatolie du Sud-Est et de l'Est durant la seconde moitié du second millénaire. Elle gagna de là l'Anatolie centrale où elle éclipsa ou assimila bon nombre de grandes déesses locales. Durant les trois premiers siècles du premier millénaire, son succès est total en Syrie du Nord et dans le Sud anatolien; les inscriptions en louvite hiéroglyphique en font l'épouse du dieu de l'orage Tarb̧unt. Au VIIe siècle av. J.-C., son culte est attesté par une inscription araméenne dans la ville de Hiérapolis-Castabala, voisine de Kybistra, que les auteurs grecs et latins désignaient comme la ville sainte de Kubaba ${ }^{28}$. À la même époque, on la voit statufiée à Boğazköy. Un peu plus tard, elle rayonne en Phrygie; un de ses hauts lieux de culte en Asie Mineure est Pessinonte. Son nom reste identique; Kubaba se retrouve dans la forme grécisée $K v \beta \eta ́ \beta \eta$ et il y beaucoup de chance que $K v \beta \varepsilon ́ \lambda \eta$ soit une forme altérée de $K v \beta \hat{\eta} \beta \eta^{29}$. Il y a continuité cultuelle dans la stabilité du nom divin et dans le maintien des caractères fondamentaux de la déesse; toutefois, sa personnalité a dû s'enrichir au cours des siècles des caractéristiques de divinités locales qu'elle absorbait, et son culte a pu évoluer, vu l'aspect «mystique» de la déesse. Par ailleurs, Hésychius signalait l'assimilation de Kubaba à Artémis; cette assertion se vérifierait à Castabala, célèbre aux dires de certains anciens par son culte à Artémis Pérasia qu'il faut probablement identifier à Kubaba ${ }^{30}$.

2. Létô : $\Lambda \eta \tau \hat{\omega}=$ lycien Lata/Lete. Je n'aborderai pas ici la problématique délicate des origines des enfants de la déesse, Apollon et Artémis; le sujet est trop complexe mais il est évident que le problème est lié à l'origine et au culte de Létô. Le but n'est pas d'assurer le triomphe d'une théorie, mais de susciter quelques réflexions. Écartons d'abord tout

28 Pour l'inscription araméenne, voir spécialement A. DUPONT-SommER et L.ROBERT, La déesse de Hiérapolis Castabala, Paris, 1964, p. 11-15. Voir aussi Strabon, Géographie , XII, 535, 537; Pline l'ancien, VI, 3, 8.

29 Cf. notamment E. LAROCHE, Koubaba, déesse anatolienne, et le problème des origines de Cybèle, in Éléments orientaux dans la religion grecque, StrasbourgParis, 1960, p. 122 et 127; C. BRIXHE, Le nom de Cybèle, in Die Sprache, 25 (1979), p. 40-45.

30 R. LEBRUN, art. cit. (n. 1), p. 87-88. 
rapprochement de Lata avec le lycien $l a d a-=\gamma v v \eta^{31}$. Observons ensuite que Létô est considérée et vénérée comme une divinité importante en Asie Mineure, en Lycie plus particulièrement, et non en Grèce continentale. La Lycie est sa patrie, Xanthos est son grand sanctuaire, tandis que Patara et Korydalla constituent des lieux de culte importants. Une tradition locale voulait de plus que Létô ait donné naissance à Apollon et Artémis à Xanthos ${ }^{32}$. Il est, par ailleurs, assuré qu'un culte à une déesse Mère / divinité des sources (louvite ?) existait depuis une haute antiquité à Xanthos. La question est ainsi de déterminer si Létô arrivant de Grèce en Asie Mineure avec ses sublimes enfants a récupéré au cours du premier millénaire le culte toujours actif de l'antique déesse louvite, comme le pense T.R. Bryce ${ }^{33}$, ou si Létô est bien une divinité indigène, avec Xanthos comme sanctuaire principal, dont le culte avec celui de ses enfants se serait introduit progressivement en Grèce via les îles. La documentation réunie à ce jour ne permet pas de trancher. Observons toutefois que, chez Homère, le trio Létô, Artémis, Apollon est manifestement du côté des Troyens, c'est-à-dire d'un peuple dont les affinités

31 Cf. R. GuSMANI, in $I F, 68$ (1963), p. 289-292. Observons cependant que tout récemment H.C. MELCHERT, Lycian Lexicon, Chapel Hill, $1993^{2}$ (Lexica Anatolica, 1), p. 38, refuse de reconnaître Létô dans le lycien Lãta; il propose pour ce terme le sens de "mort", mais voyant dans $L e \theta \theta_{l}(T L 44$ b 61$)<*$ Lete$h i$ - le nom de Létô, ce qui suppose le thème Lete-.

32 Cf. l'inscription des Kyténiens trouvée à Xanthos (inéd. partiellement) (205 av.

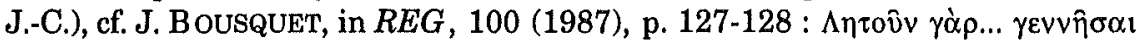

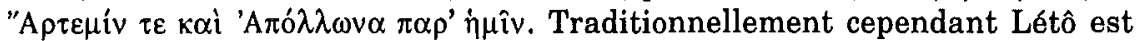
censée avoir donné naissance à Artémis et Apollon soit à Ortygie, soit surtout dans l'île de Délos, d'où elle gagna la Lycie, en particulier les rives du Xanthos et les eaux lustrales de la source Mélitè (cf. l'adjectif louvite maliddu «doux") où elle voulait baigner ses prestigieux enfants. Cf. ANTONINUS LiBERALIS, Mét., 35; Ovide, Mét., VI, 317-381; pour Mélitè, cf. G. Neumann, Beiträge zu Lykischen, in Die Sprache, 7 (1961), p. 73-76. Ajoutons que Létô était aussi vénérée à Oinoanda de Lycie (la Wiyanawanda du second millénaire) d'après des inscriptions trouvées entre 1974 et 1977 et entreposées aujourd'hui au musée de Fethiye, cf. A. HaLL, in Anat. St. , 27 (1977), p. 193 sq; le culte est aussi attesté à Olymos d'après les inscriptions 895-897 détaillant l'inventaire du temple de Létô (seconde moitié du IIe siècle av. J.-C.) : W. BLÜMEL, in EA, 13 (1989), p. 7-9.

33 T.R. BRYCE, The Arrival of the Goddess Letô in Lycia , in Historia, 32 (1983), p. 1-13. Sur cette question, il n'est pas inutile de consulter W. BURKERT, Apellai und Apollon, in RhM, 118 (1975), p. 1-21. 
ethniques avec les Louvites semblent de plus en plus évidentes et qui se voit menacé par l'expansionnisme achéen ${ }^{34}$.

\section{Les «Champs Élysées», un cas de continuité et d'emprunt}

Au sein des diverses croyances émaillant la «religion grecque» s'est développée, face au pessimisme généralisé concernant l'Hadès, une lueur d'espoir avec l'introduction du concept des «Champs Élysées» $=$ 'H $\lambda \hat{\sigma} \sigma ı v \pi \varepsilon \delta$ íov. Il convient de s'interroger sur l'origine spatiotemporelle de cette croyance. La question reste obscure, mais il n'est pas impossible qu'elle ait pénétré le monde grec au départ de l'Asie Mineure / Anatolie, comme le pensait H. Wagenvoort ${ }^{35}$. Au point de départ de la réflexion, ne perdons pas de vue que l'accès à ce lieu de félicité est réservé à des gens de "Qualité». Que signifie maintenant le terme 'H $\lambda$ v́otos? Plusieurs spécialistes se sont penchés récemment sur le problème et sont arrivés à d'intéressantes hypothèses. Ainsi, ne pourrait-on, comme ce fut suggéré en 1969 par J. Puhvel et en 1991 par F. Bader, reconnaître dans 'H $\lambda$ v́oı́s la grécisation du hittite Wilusiya (Wilusa), ce toponyme d'Anatolie occidentale plusieurs fois mentionné dans les tablettes hittites et dont la signification serait «fait de prairie» si l'on y reconnaît avec d'autres un dérivé du hittite wellu- «prairie» ${ }^{*} w e l-n u^{36}$. Dès lors, 'H $\mathrm{\lambda}$ v́oı $\pi \varepsilon \delta$ íov signifie «plaine faite de prairie» perçue comme un lieu de bien-être pour l'au-delà. L'interprétation de 'H $\lambda$ v́o los à partir des faits hittites prend d'autant plus de relief si l'on songe que le roi hittite divinisé à sa mort était censé faire paître les troupeaux du dieu Soleil dans une sorte de prairie idéale ${ }^{37}$.

34 Ainsi, les noms propres troyens s'avèrent souvent anatoliens (louvites) : un bon exemple en est le nom même du roi Priam <étymon louvite *Pariya-muwa "super flux vital». D'autre part, les alliés des Troyens ne se comptent-ils pas parmi les Lyciens et Ciliciens, donc des populations relevant du groupe louvite?

35 H. WAGENVOORT, The Journey of the Souls of the Dead to the Isles of the Blessed, in Mnemosyne, 24 (1971), p. 113-161.

36 Voir J. PUHVEL, in $K Z, 83$ (1969), p. 64-69; F. BADER, in $B S L, 86$ (1991), p. 127. L'étymologie proposée par ces deux savants est aussi partagée par J.J.S. W EITENBERG, Die hethitischen U-Stämme, Amsterdam, 1984, p. 182-183, de

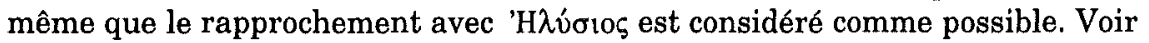
aussi R. STEFANINI, in Paideia, 29 (1975), p. 257-267.

37 Cf. M. VIEYRA, Ciel et enfers hittites, in RA, 59 (1965), p. 127-130. 
Le présent exposé s'est limité à quelques exemples significatifs et, d'emblée, on constatera que la frontière entre syncrétisme et assimilation est souvent difficile à établir. Cependant, durant la période grécoasianique, Héraklès et Hermès se prêtèrent remarquablement à des formes de syncrétisme avec Santa-Melqart, l'Hermès de Korykos avec le dieu local protecteur de la nature sauvage; l'Arès et les Dioscures asianiques peuvent aussi présenter un aspect syncrétique avec leurs homologues grecs, à moins qu'il ne s'agisse d'une pure assimilation. Cette dernière est évidente dans le chef de Maliya assimilée à Athéna et dans celui des dieux de l'orage anatoliens (type Tarbunt) assimilés à Zeus, encore qu'il existe un type de Zeus karpodotès (tout comme un Arès karpodotès) issu, en Anatolie méridionale centrale et orientale, de la continuité d'un syncrétisme opéré au début du premier millénaire entre le Baal phénicien (type du Baal ougaritique) et le Tarbunt louvite. De façon générale, les Zeus asianiques recouvrent les dieux de l'orage locaux. Kubèbè-Cybèle paraît offrir aussi des aspects syncrétiques.

Face au puissant mouvement d'hellénisation de l'Asie Mineure, des figures divines locales se sont maintenues telles quelles, principalement en Lycie, mais aussi en Carie, en Pisidie et en Pamphylie, et, probablement en bien d'autres secteurs méridionaux. Cette situation se vérifie surtout en des lieux tenus à l'écart des grandes voies de pénétration ou sur des sites naturellement protégés. Les cariens Sinuri, Arkésima et Basileus Caunios, Kakasbos (néanmoins parfois assimilé à Héraklès), Tobaloas ou encore Trôsobios illustrent magnifiquement cette continuité religieuse anatolienne interrompue de façon sensible par les progrès du christianisme. La Lycie constitue la région où la tradition anatolienne-louvite a le mieux résisté.

Institut Catholique de Paris

R. LEBRUN

Rue d'Assas, 21

F - 75270 PARIS Cedex 06 